\title{
TRANSCENDENTAL MEROMORPHIC SOLUTIONS OF SOME ALGEBRAIC DIFFERENTIAL EQUATIONS
}

\author{
KATSUYA ISHIZAKI ${ }^{\oplus}$ and NOBUSHIGE TODA
}

(Received 6 December 2005; revised 16 May 2006)

Communicated by P. Fenton

\begin{abstract}
In this paper we treat transcendental meromorphic solutions of some algebraic differential equations. We consider the number of distinct transcendental meromorphic solutions. Algebraic relations between meromorphic solutions and comparisons of the growth of transcendental meromorphic solutions are also discussed.
\end{abstract}

2000 Mathematics subject classification: primary 34A20; secondary 30D35.

\section{Introduction}

The binomial differential equation

$$
\left(y^{\prime}\right)^{n}=R(z, y)
$$

where $n$ is a positive integer and $R(z, y)$ is a rational function in $z$ and $y$, has been studied under the assumption that it has a transcendental meromorphic solution $y$ in the complex plane (for example, Yosida [17], Laine [10]). The result due to Steinmetz [13], Bank and Kaufman [2] states that by a suitable Möbius transformation $v=(\alpha y+\beta) /(\gamma y+\delta)$, where $\alpha \delta-\beta \gamma \neq 0$, the binomial equation is classified into

Grant-in-Aid for Scientific Research, Japan Society for the Promotion of Science, (C) (1)

(No. 16540202)

(C) 2007 Australian Mathematical Society 1446-7887/07 \$A2.00+0.00 
the following six simple differential equations:

$$
\begin{aligned}
v^{\prime} & =a_{2}(z) v^{2}+a_{1}(z) v+a_{0}(z) \\
\left(v^{\prime}\right)^{2} & =a(z)(v-b(z))^{2}\left(v-\tau_{1}\right)\left(v-\tau_{2}\right) \\
\left(v^{\prime}\right)^{2} & =a(z)\left(v-\tau_{1}\right)\left(v-\tau_{2}\right)\left(v-\tau_{3}\right)\left(v-\tau_{4}\right) \\
\left(v^{\prime}\right)^{3} & =a(z)\left(v-\tau_{1}\right)^{2}\left(v-\tau_{2}\right)^{2}\left(v-\tau_{3}\right)^{2} \\
\left(v^{\prime}\right)^{4} & =a(z)\left(v-\tau_{1}\right)^{2}\left(v-\tau_{2}\right)^{3}\left(v-\tau_{3}\right)^{3} \\
\left(v^{\prime}\right)^{6} & =a(z)\left(v-\tau_{1}\right)^{3}\left(v-\tau_{2}\right)^{4}\left(v-\tau_{3}\right)^{5}
\end{aligned}
$$

where $\tau_{1}, \ldots, \tau_{4}$ are distinct constants and $a_{j}(z)(\not \equiv 0), j=0,1,2, a(z), b(z)$ are rational functions. The result of Steinmetz cited above [13, Theorem 2], was generalized by v. Rieth [16] and He-Laine [7] to cover the case when $R(z, y)$ is rational in $y$ with meromorphic coefficients.

Throughout this paper 'meromorphic' means 'meromorphic in the complex plane' and we use the standard notation of the Nevanlinna theory of meromorphic functions (see for example, $[6,11,12]$ ).

We consider the following three problems for the equations (1.2) and (1.3). For equation (1.2) we especially consider the case when $b(z)$ is a constant and we refer to this case as $\left(1.2^{*}\right)$, The equations $\left(1.2^{*}\right)$ and (1.3) are treated in Section 2 and in Section 3 respectively.

The first problem is to classify the equations by the number of transcendental meromorphic solutions. The differential equations (1.1)-(1.6) do not always admit transcendental meromorphic solutions. It depends on the coefficients of the equations. We investigate how many transcendental meromorphic solutions the differential equations have and under what conditions they have an infinite number of transcendental meromorphic solutions. Some results are already known about the number of meromorphic solutions to the Ricatti equation (1.1) (see, for example, [1], or [11, Chapter 9]). Answers to this problem for $\left(1.2^{*}\right)$ are given in Corollary 2.4 and for (1.3) are given in parts (a) and (b) of Corollary 3.2.

The second problem is to find algebraic relations between meromorphic solutions. For the case of the Riccati equation (1.1), four distinct solutions $f_{1}, f_{2}, f_{3}, f_{4}$ of (1.1) satisfy $\mathscr{R}\left(f_{1}, f_{2}, f_{3}, f_{4}\right)=c$, for a constant $c$, where $\mathscr{R}$ is a cross ratio of four elements (see, for example, [9, Section 4.2]). We shall give an answer for $\left(1.2^{*}\right)$ by proving Theorem 2.1, and give an answer for (1.3) by proving Theorem 3.1 (iii).

The third problem is to compare the growth of transcendental meromorphic solutions. There are many results on the growth of transcendental meromorphic solutions of these six differential equations (see, for example, $[2,13,14]$ ). The fact proved in [2] and in [14] is that for the transcendental meromorphic solutions $f$ of $\left(1.2^{*}\right)$ or (1.3), the order of $f$ is a positive integral multiple of $1 / 2$, which is dependent on the coefficients of the equation. For example (cf. [14, Satz 1]), for any solution 
of $\left(f^{\prime}\right)^{2}=A(z)\left(f^{2}-1\right)$ the order of $f$ is equal to $1+d / 2$ where $d \geq-1$ and

$$
A(z)=c_{1} z^{d}+c_{2} z^{d-1}+\cdots \text { for } z \rightarrow \infty, c_{1} \neq 0 .
$$

This says that for given (fixed) coefficients, all transcendental meromorphic solutions $f$ and $g$ of the equation have the same order of growth.

We shall give more detailed estimates of growth for transcendental meromorphic solutions of $\left(1.2^{*}\right)$ in Theorem 2.1 and of (1.3) in part (c) of Corollary 3.2.

\section{Results for the equation (1.2)}

This section is devoted to answering the question, which we posed in Section 1 for the equation $\left(1.2^{*}\right)$ (that is, equation (1.2) in the case where $b(z)$ is a constant).

The linear transformation

$$
f=\frac{2\left(\tau_{2}-b\right)\left(v-\tau_{1}\right)}{\left(\tau_{2}-\tau_{1}\right)(v-b)}-1
$$

can be used to transform equation $\left(1.2^{*}\right)$ into

$$
\left(f^{\prime}\right)^{2}=A(z)\left(f^{2}-1\right),
$$

where $A(z)=\left(b-\tau_{1}\right)\left(b-\tau_{2}\right) a(z)$. This form of the equation is more suitable for investigation of solutions. We denote by $\mathfrak{S}(A)$ the set of transcendental meromorphic solutions of (2.1) for a given rational function $A$, and denote by $\# \mathfrak{S}(A)$ the number of functions in $\mathfrak{S}(A)$.

In this section we prove the following theorems and corollaries.

THEOREM 2.1. Suppose that the differential equation (2.1) possesses distinct transcendental meromorphic solutions $f$ and $g$. Then there is a constant $c$ such that

$$
f^{2}+2 c f g+g^{2}=1-c^{2} .
$$

Conversely, if there are two nonconstant meromorphic functions $f$ and $g$ satisfying (2.2), then the following relation holds:

$$
\frac{\left(f^{\prime}\right)^{2}}{f^{2}-1}=\frac{\left(g^{\prime}\right)^{2}}{g^{2}-1}
$$

so that if $f$ is a solution of (2.1) then so is $g$.

COROLLARY 2.2. Suppose that the differential equation (2.1) possesses transcendental meromorphic solutions $f$ and $g$. Then we have

$$
T(r, g)=T(r, f)+O(1) .
$$


THEOREM 2.3. Suppose that the differential equation (2.1) admits at least three transcendental meromorphic solutions. Then the following statements are true.

(i) There is a rational function $\alpha(z)$ such that $A(z)=\alpha(z)^{2}$.

(ii) We can write $\alpha(z)$ in (i) as a decomposition of partial fractions

$$
\alpha(z)=p(z)+\sum_{j=1}^{n} k_{j}\left(z-\tau_{j}\right)^{-1},
$$

where $p(z)$ is a polynomial not identically equal to $0, k_{j}(j=1, \cdots, n)$ are integers and $\tau_{j}(j=1, \ldots, n)$ are distinct constants. Moreover, for any transcendental meromorphic solution $f$ there exists a constant $C \in \mathbb{C}$ such that

$$
f(z)=\cosh \left(\int_{0}^{z} p(z) d z+\sum_{j=1}^{n} \log \left(z-\tau_{j}\right)^{k_{j}}+C\right) .
$$

COROLLARY 2.4. (a) If the differential equation (2.1) admits at least three transcendental meromorphic solutions then $\# \mathfrak{S}(A)=\infty$.

(b) For a rational function $A$, the number of transcendental meromorphic solutions of (2.1) is 0,2 or $\infty$.

We note that any nonconstant meromorphic solution $f$ of (2.1) satisfies the second order linear differential equation

$$
f^{\prime \prime}-\left(\frac{A^{\prime}}{2 A}\right) f^{\prime}-A f=0 .
$$

In fact, differentiating (2.1), we have $2 f^{\prime} f^{\prime \prime}=A^{\prime}\left(f^{2}-1\right)+2 A f f^{\prime}$. Combining this with (2.1), we obtain (2.7) since $f^{\prime} \neq 0$.

For the proofs of Theorems 2.1 and 2.3 we need some lemmas given below.

LEMMA 2.5. [5, Theorem 1] Let $F$ and $G$ be meromorphic functions. $F$ and $G$ satisfy $F^{2}+G^{2}=1$ if and only if there is a meromorphic function $\beta(z)$ such that

$$
F=\frac{2 \beta}{1+\beta^{2}} \text { and } G=\frac{1-\beta^{2}}{1+\beta^{2}}
$$

LEMMA 2.6. Let $f$ be a nonconstant meromorphic function and put

$$
R(z)=\frac{\left(f^{\prime}\right)^{2}}{f^{2}-1}
$$

If $R(z)$. has poles then any pole of $R(z)$ is of order at most 2 . 
PROOF. Any pole $z_{0}$ of $R(z)$ is either a pole of $f$, a zero of $f(z)-1$ or a zero of $f(z)+1$. If $z_{0}$ is a pole of $f$ then a standard pole order comparison of (2.8) implies that $R(z)$ has a double pole at $z_{0}$. By similar reasoning, if $f(z)= \pm 1+\sum_{j=k}^{\infty} \alpha_{j}\left(z-z_{0}\right)^{j}$ around $z_{0}$ then $R(z)$ is regular at $z_{0}$ when $k \geq 2$, while $R(z)$ has a simple pole at $z_{0}$ when $k=1$.

LEMMA 2.7. Suppose that a meromorphic function $\alpha$ can be expressed in a neighbourhood of $a_{0}$ as

$$
\alpha(z)=\frac{k}{z-a_{0}}+h(z), \quad(k \neq 0),
$$

where $h(z)$ is regular at $a_{0}$. Then, the differential equation

$$
w^{\prime \prime}-\left(\frac{\alpha^{\prime}(z)}{\alpha(z)}\right) w^{\prime}-\alpha^{2}(z) w=0
$$

has a single-valued meromorphic solution in a neighbourhood of $a_{0}$ if and only if $k$ is an integer.

PROOF. From (2.9), it is easy to see that $a_{0}$ is a regular-singular point for (2.10) (see [8, Satz 3.2]). The corresponding indicial equation at $a_{0}$ is

$$
\rho(\rho-1)+\rho-k^{2}=\rho^{2}-k^{2}=0
$$

and its solutions are $\rho=k$ and $\rho=-k$. Therefore it is easy to see that (2.10) has a nonconstant meromorphic solution in a neighbourhood of $a_{0}$ if and only if $k$ is an integer.

PROOF OF THEOREM 2.1. Assume that $f$ and $g$ are transcendental meromorphic solutions to $(2.1)$, so that

$$
\left(f^{\prime}\right)^{2}=A\left(f^{2}-1\right) \text { and }\left(g^{\prime}\right)^{2}=A\left(g^{2}-1\right) .
$$

Then it follows from (2.7) that

$$
f^{\prime \prime}-\left(\frac{A^{\prime}}{2 A}\right) f^{\prime}-A f=0 \text { and } g^{\prime \prime}-\left(\frac{A^{\prime}}{2 A}\right) g^{\prime}-A g=0 .
$$

We add the two equations in (2.12) and then multiply the obtained equality by $2\left(f^{\prime}+g^{\prime}\right) / A$ to obtain

$$
\frac{2\left(f^{\prime}+g^{\prime}\right)\left(f^{\prime \prime}+g^{\prime \prime}\right)}{A}-\frac{A^{\prime}}{A^{2}}\left(f^{\prime}+g^{\prime}\right)^{2}=2(f+g)\left(f^{\prime}+g^{\prime}\right),
$$

from which we have $\left(\left(f^{\prime}+g^{\prime}\right)^{2} / A\right)^{\prime}=\left((f+g)^{2}\right)^{\prime}$ and hence

$$
\frac{\left(f^{\prime}+g^{\prime}\right)^{2}}{A}=(f+g)^{2}+c^{\prime}
$$


where $c^{\prime}$ is a constant. From (2.11) and (2.13) we eliminate $A, f^{\prime}$ and $g^{\prime}$ to obtain (2.2), where $c=1+c^{\prime} / 2$.

To prove the converse statement in the theorem, we suppose that two nonconstant meromorphic functions $f$ and $g$ satisfy (2.2). When $c^{2}=1$, we have $f= \pm g$ and so the relation (2.3) holds. We consider the case $c^{2} \neq 1$. Write (2.2) as

$$
(f+c g)^{2}+\left(1-c^{2}\right) g^{2}=1-c^{2} .
$$

Differentiating both sides of (2.14), we have

$$
\left(f^{\prime}+c g^{\prime}\right)(f+c g)+\left(1-c^{2}\right) g^{\prime} g=0 .
$$

Combining (2.14) and (2.15), we obtain

$$
\frac{\left(g^{\prime}\right)^{2}}{1-g^{2}}=\frac{\left(f^{\prime}+c g^{\prime}\right)^{2}}{\left(1-c^{2}\right) g^{2}}
$$

Similarly we obtain by symmetry

$$
\frac{\left(f^{\prime}\right)^{2}}{1-f^{2}}=\frac{\left(g^{\prime}+c f^{\prime}\right)^{2}}{\left(1-c^{2}\right) f^{2}}
$$

We can write (2.15) as $f\left(f^{\prime}+c g^{\prime}\right)=-g\left(g^{\prime}+c f^{\prime}\right)$, so that the right-hand sides of (2.16) and (2.17) are equal, which proves that $f$ and $g$ satisfy (2.3).

PROOF OF COROLLARY 2.2. If $c^{2}=1$, then $f= \pm g$ and we have $T(r, f)=T(r, g)$. Hence 'we only treat the case $c^{2} \neq 1$. From (2.2), we have

$$
\left(\frac{f}{g}\right)^{2}+\frac{2 c f}{g}+1=\frac{1-c^{2}}{g^{2}}
$$

from which we have, by Nevanlinna's first fundamental theorem,

$$
2 T(r, g)=2 T(r, f / g)+O(1) \text {. }
$$

Changing the roles of $f$ and $g$ and using Nevanlinna's first fundamental theorem, we obtain the relation $2 T(r, f)=2 T(r, g / f)+O(1)=2 T(r, f / g)+O(1)$. Combining the two relations above, we obtain (2.4).

PROOF OF THEOREM 2.3(i). By the hypothesis of this theorem and by Theorem 2.1, there are transcendental meromorphic functions $f$ and $g$ satisfying

$$
f^{2}+2 c f g+g^{2}=1-c^{2} \quad\left(c^{2} \neq 1\right),
$$

from which we have $f^{2}+\left((c f+g) /\left(\sqrt{1-c^{2}}\right)\right)^{2}=1$. By Lemma 2.5 , there is a meromorphic function $\beta(z)$ such that $f=2 \beta /\left(1+\beta^{2}\right)$. We see that because $f$ is transcendental, so is $\beta$. Hence

$$
A(z)=\frac{\left(f^{\prime}\right)^{2}}{f^{2}-1}=-\left(\frac{2 \beta^{\prime}}{1+\beta^{2}}\right)^{2}=\left(\frac{2 i \beta^{\prime}}{1+\beta^{2}}\right)^{2} .
$$


That is to say, $A(z)=\alpha(z)^{2}$ where $\alpha(z)=\left(2 i \beta^{\prime}\right) /\left(1+\beta^{2}\right)$. Since $A(z)$ is a rational function, $\alpha(z)$ must be a rational function.

PROOF OF THEOREM 2.3(ii). By Theorem 2.3 (i), we can write $A(z)=\alpha(z)^{2}$ for a rational function $\alpha(z)$. If $\alpha(z)$ has a pole, then the pole is simple by Lemma 2.6 and the residue at the pole must be an integer by Lemma 2.7 . Hence we can write $\alpha(z)$ in the form

$$
\alpha(z)=p(z)+\sum_{j=1}^{n} k_{j}\left(z-\tau_{j}\right)^{-1},
$$

where $p(z)$ is a polynomial, $n$ is the number of poles of $\alpha(z), k_{j}(j=1, \ldots, n)$ are integers, and $\tau_{j}(j=1, \ldots, n)$ are distinct constants. If we put $\zeta(z)=\int_{0}^{z} p(t) d t$ then the meromorphic functions

$$
f_{1}(z)=e^{\zeta(z)} \prod_{j=1}^{n}\left(z-\tau_{j}\right)^{k_{j}} \quad \text { and } \quad f_{2}(z)=e^{-\zeta(z)} \prod_{j=1}^{n}\left(z-\tau_{j}\right)^{-k_{j}},
$$

which are linearly independent, satisfy the linear differential equation (2.10). Since any solution $f(z)$ of (2.1) satisfies (2.10), $f(z)$ can be expressed as a linear combination of $f_{1}$ and $f_{2}$, say

$$
f(z)=C_{1} f_{1}(z)+C_{2} f_{2}(z),
$$

where $C_{1}$ and $C_{2}$ are constants. As $f_{1}^{\prime}(z)=\alpha(z) f_{1}(z), f_{2}^{\prime}(z)=-\alpha(z) f_{2}(z)$ and $f_{1} f_{2}=1$ from (2.18), by substituting (2.19) into (2.1), we obtain that $C_{1} C_{2}=1 / 4$. Therefore we see that for some $C \in \mathbb{C}, f(z)$ is represented in the form

$$
f(z)=\cosh \left(\zeta(z)+\sum_{j=1}^{n} \log \left(z-\tau_{j}\right)^{k_{j}}+C\right) .
$$

It is immediately concluded that if $p(z) \equiv 0$ then meromorphic solutions to (2.1) are rational functions, which is a contradiction. Hence $p(z) \not \equiv 0$ and the assertion follows.

PROOF OF COROLLARY 2.4. (a) It follows from the proof of Theorem 2.3 (ii) that if $A=\alpha^{2}$, where $\alpha$ satisfies (2.5), then a meromorphic function of the form (2.6) is a solution of (2.1). This implies that $\mathfrak{S}(A)$ is an uncountable set when $p(z) \not \equiv 0$. This implies that if (2.1) possesses at least three distinct transcendental meromorphic solutions then $\# \mathfrak{S}(A)=\infty$.

(b) It is clear that if $f$ is a transcendental meromorphic solution of (2.1) then $-f$ is also a transcendental meromorphic solution of (2.1). By part (a), \#SS $(A) \geq 3$ implies $\# \mathfrak{S}(A)=\infty$. Therefore we have proved (b). 
REMARK 1. We mention a condition which implies $\mathfrak{S}(A)$ is an empty set: if $A$ has at least one pole of order not less than 3 then $\# \mathfrak{S}(A)=0$. This is a direct consequence of Lemma 2.6.

PRoOF. We proved part (a) of Corollary 2.4 by means of Theorem 2.3. We mention here that we can get the same result by only using the algebraic relation (2.2) and the relation (2.4). In fact, by the hypothesis of this Corollary, there are two meromorphic functions $f$ and $g$ in $\mathfrak{S}(A)$ satisfying $f^{2}+2 c f g+g^{2}=1-c^{2}\left(c^{2} \neq 1\right)$, from which we have

$$
f^{2}+\left(\frac{c f+g}{\sqrt{1-c^{2}}}\right)^{2}=1
$$

This shows that $(c f+g) / \sqrt{1-c^{2}} \in \mathfrak{S}(A)$ by (2.3) in Theorem 2.1 and (2.4) since $f \in \mathfrak{S}(A)$. Put

$$
h=\frac{c f+g}{\sqrt{1-c^{2}}} \text { and } F=\gamma f+\delta h
$$

where $\gamma$ and $\delta$ are constants satisfying $\gamma^{2}+\delta^{2}=1$. Then

$$
f^{2}+h^{2}=1 \text { and } f f^{\prime}=-h h^{\prime} .
$$

Now we are going to prove that $F \in \mathfrak{S}(A)$. In fact, by (2.20),

$$
\begin{aligned}
\left(F^{\prime}\right)^{2} & =\gamma^{2}\left(f^{\prime}\right)^{2}+2 \gamma \delta f^{\prime} h^{\prime}+\delta^{2}\left(h^{\prime}\right)^{2} \\
& =\gamma^{2} A\left(f^{2}-1\right)+\delta^{2} A\left(h^{2}-1\right)-\frac{2 \gamma \delta f\left(f^{\prime}\right)^{2}}{h} \\
& =A\left(\gamma^{2} f^{2}+\delta^{2} h^{2}-1\right)+2 \gamma \delta A f h=A\left((\gamma f+\delta h)^{2}-1\right)
\end{aligned}
$$

since $\left(f^{\prime}\right)^{2} / h^{2}=\left(h^{\prime}\right)^{2} / f^{2}=-A$ by (2.20) and $f, h \in \mathfrak{S}(A)$. It follows from (2.21) that $F=\gamma f+\delta h$ is a meromorphic solution of (2.1) and by (2.4) that $\gamma f+\delta h \in \mathbb{S}(A)$. This proves the assertion.

\section{Results for the equation (1.3)}

In this section we are concerned with the differential equation of the type (1.3) in Section 1. It will be seen below that solutions of the equation (1.3) are closely connected with the Weierstrass $\wp$-function. We choose and fix a $\wp$-function satisfying

$$
\left(\wp^{\prime}\right)^{2}=4 \wp^{3}-\tilde{g}_{2 \wp} \wp-\tilde{g}_{3}
$$


where $\tilde{g}_{2}, \tilde{g}_{3}$, are constants satisfying $27 \tilde{g}_{3}^{2}-\tilde{g}_{2}^{3} \neq 0$. For the sake of brevity we put $G(x)=4 x^{3}-\tilde{g}_{2} x-\tilde{g}_{3}$, and we denote by $e_{1}, e_{2}, e_{3}$ the distinct roots of $G(x)=0$. For any solution $v$ of (1.3), we set

$$
\begin{aligned}
f(z) & =\frac{\alpha}{v(z)-\tau_{4}}-\beta \quad \text { with } \quad \alpha=-\frac{\left(\tau_{1}-\tau_{4}\right)\left(\tau_{2}-\tau_{4}\right)\left(\tau_{3}-\tau_{4}\right)}{4}, \\
\beta & =\frac{1}{12}\left(2 \tau_{4}\left(\tau_{1}+\tau_{2}+\tau_{3}\right)-\left(\tau_{1} \tau_{2}+\tau_{2} \tau_{3}+\tau_{3} \tau_{1}\right)-3 \tau_{4}^{3}\right) .
\end{aligned}
$$

Then the equation of type (1.3) can be translated into the form

$$
\left(f^{\prime}\right)^{2}=A(z)\left(4 f^{3}-\tilde{g}_{2} f-\tilde{g}_{3}\right)=A(z) G(f),
$$

where $A(z) \not \equiv 0$ is a rational function. We denote by $\mathfrak{T}(A)$ the set of transcendental meromorphic solutions of (3.2) for a given rational function $A$, and denote by $\# T(A)$ the number of functions in $\mathfrak{T}(A)$.

The purpose of this section is to prove the following theorem and corollary.

THEOREM 3.1. Suppose that the equation (3.2) admits two transcendental meromorphic solutions $f$ and $g$ such that $f \neq L(g)$ for some Möbius transformation $L$ such that $L(z) \neq \equiv z$. Then the following statements are true.

(i) There exists a polynomial $a(z)$ such that $A(z)=a^{\prime}(z)^{2}$.

(ii) Any $f(z) \in \mathfrak{T}(A)$ can be expressed as

$$
f(z)=\wp(a(z)+c), \quad c \in \mathbb{C},
$$

where $\wp$ is the Weierstrass $\wp$ function given in (3.1).

(iii) Let $u(z)$ and $v(z)$ denote arbitrary distinct transcendental meromorphic solutions of (3.2). Then there exists a constant $d_{0} \in \mathbb{C}$, such that $U=u-d_{0}$ and $V=v-d_{0}$ satisfy an algebraic relation

$$
U^{2} V^{2}-G_{2} U V-G_{1}(U+V)-G_{0}=0,
$$

where $G_{0}, G_{1}$ and $G_{2}$ are constants.

Conversely, if transcendental meromorphic functions $U$ and $V$ satisfy (3.4) then we have

$$
\frac{\left(U^{\prime}\right)^{2}}{K(U)}=\frac{\left(V^{\prime}\right)^{2}}{K(V)}
$$

where $K(x)$ is a polynomial of degree 3 expressed as

$$
K(x)=4 x^{3}+\left(\frac{G_{0}+G_{2}^{2}}{G_{1}}\right) x^{2}+2 G_{2} x+G_{1} .
$$


COROLLARY 3.2. (a) If the equation (3.2) admits two transcendental meromorphic solutions $f$ and $g$ such that $f \neq L(g)$ for some Möbius transformation $L$ which is not the identity, then $\# \mathbb{T}(A)=\infty$.

(b) For a rational function $A$, the number of transcendental meromorphic solutions of (3.2) is 0,4 or $\infty$.

(c) For any transcendental meromorphic solutions $f$ and $g$ of (3.2) we have

$$
T(r, g)=T(r, f)+S(r),
$$

where $S(r)$ is small with respect to $T(r, f)$ and $T(r, g)$.

We need the following results due to Bank and Kaufman [2, Lemma 5], and Valiron [15].

LEMMA 3.3. Let $H(w)$ be a polynomial having constant coefficients, and let $w(z)$ be a nonconstant elliptic function of elliptic order $q$ which is a solution of the differential equation $\left(w^{\prime}\right)^{q}=H(w)$. Then the following statements are true.

(a) If $c_{0}$ and $c_{1}$ are complex numbers satisfying $c_{1}^{q}=H\left(c_{0}\right)$ then there exists a complex number $\zeta$ such that $w(\zeta)=c_{0}$ and $w^{\prime}(\zeta)=c_{1}$.

(b) Any solution of the differential equation $\left(w^{\prime}\right)^{q}=H(w)$ which is meromorphic and nonconstant in a region of the plane must be of the form $w(z+C)$ where $C$ is a constant.

The lemma given below is also needed for the proof of Theorem 3.1.

LEMMA 3.4. Suppose that (3.2) has distinct transcendental meromorphic solutions $f$ and $g$. If $f$ and $g$ have a common pole $z_{0}$ and we let $\varphi=f-g$ then $\varphi$ does not have a zero at $z_{0}$.

Proof. We write $A$ in a neighbourhood of $z_{0}$ as

$$
A(z)=R_{A}\left(z-z_{0}\right)^{\lambda}+O\left(z-z_{0}\right)^{\lambda+1}, \quad R_{A} \neq 0,
$$

where $\lambda$ is an integer. Let $\mu_{f}$ and $\mu_{g}$ denote the orders of the poles of $f$ and $g$ at $z_{0}$. From (3.2), we have $-2\left(\mu_{f}+1\right)=\lambda-3 \mu_{f}$, so $\mu_{f}=2+\lambda$. Similarly we have $\mu_{g}=2+\lambda$. For the sake of brevity we write $\mu_{f}=\mu_{g}=\mu$.

Write $f$ and $g$ in a neighbourhood of $z_{0}$ as

$$
\begin{aligned}
& f(z)=\frac{R_{f}}{\left(z-z_{0}\right)^{\mu}}+O\left(z-z_{0}\right)^{-(\mu-1)}, \quad R_{f} \neq 0, \\
& g(z)=\frac{R_{g}}{\left(z-z_{0}\right)^{\mu}}+O\left(z-z_{0}\right)^{-(\mu-1)}, \quad R_{g} \neq 0 .
\end{aligned}
$$


Substituting these representations into (3.2) and comparing the coefficients of terms $\left(z-z_{0}\right)^{-2(\mu+1)}$, we obtain

$$
R_{f}=R_{g}=\frac{\mu^{2}}{4 R_{A}}
$$

It follows from (3.2) that

$$
\left(\frac{\varphi^{\prime}}{\varphi}\right)\left(f^{\prime}+g^{\prime}\right)=A\left(4\left(f^{2}+f g+g^{2}\right)-\tilde{g}_{2}\right) .
$$

Assume that $\varphi$ has a zero at $z_{0}$ of order $\sigma>0$. We compare the coefficients of $\left(z-z_{0}\right)^{-(\mu+2)}$ in the Laurent expansions in both sides of (3.12). Using (3.11), we obtain

$$
\sigma\left(-\frac{\mu^{3}}{4 R_{A}}-\frac{\mu^{3}}{4 R_{A}}\right)=R_{A}\left(4\left(\frac{\mu^{4}}{16 R_{A}^{2}}+\frac{\mu^{4}}{16 R_{A}^{2}}+\frac{\mu^{4}}{16 R_{A}^{2}}\right)\right),
$$

which implies $-\sigma=3 \mu / 2$, which is absurd. We have thus proved Lemma 3.4.

The following remark states some basic properties of solutions of (3.2).

REMARK 2. (A) Every solution $f$ of (3.2) satisfies

$$
f^{\prime \prime}=\frac{A^{\prime}(z)}{2 A(z)} f^{\prime}+\frac{A(z)}{2}\left(12 f^{2}-\tilde{g}_{2}\right) .
$$

Moreover, if $f$ and $g$ are distinct solutions of (3.2), then we have

$$
\begin{aligned}
& \varphi^{\prime \prime}-\frac{A^{\prime}(z)}{2 A(z)} \varphi^{\prime}-6 A(z)(f+g) \varphi=0, \quad \text { or } \\
& \frac{\varphi^{\prime \prime}}{\varphi}-\frac{A^{\prime}(z)}{2 A(z)} \frac{\varphi^{\prime}}{\varphi}=6 A(z)(f+g),
\end{aligned}
$$

where $\varphi=f-g$.

(B) Let $f$ be a transcendental meromorphic solution of (3.2). We introduce here the following four Möbius transformations:

$$
\begin{array}{ll}
L_{0}(x)=x, & L_{1}(x)=\frac{e_{1} x+e_{1}^{2}-e_{2}^{2}-e_{1} e_{2}}{x-e_{1}}, \\
L_{2}(x)=\frac{e_{2} x+e_{2}^{2}-e_{3}^{2}-e_{2} e_{3}}{x-e_{2}}, & L_{3}(x)=\frac{e_{3} x+e_{3}^{2}-e_{1}^{2}-e_{3} e_{1}}{x-e_{3}} .
\end{array}
$$

We see that $L_{j}(f)(j=0,1,2,3)$ are also solutions of (3.2), which is verified by direct computations. Moreover, we assert that for any other Möbius transformation $L(x)=(a x+b) /(c x+d)$, with $\Delta=a d-b c \neq 0$, the equation (3.2) is not satisfied by $L(f)$. To show this, we assume that $L(f)$ satisfies (3.2), that is,

$$
\Delta^{2} \frac{\left(f^{\prime}\right)^{2}}{(c f+d)^{4}}=A(z)\left(4\left(\frac{a f+b}{c f+d}\right)^{3}-\tilde{g}_{2}\left(\frac{a f+b}{c f+d}\right)-\tilde{g}_{3}\right) \text {. }
$$


First we treat the case $c=0$. In this case we may assume that $d=1$ and $a \neq 0$. Using (3.2) and (3.16), we eliminate $f^{\prime}$ and obtain a polynomial in $f$ which must vanish. Then we have that $a=1$ and $b=0$ since $f$ is a transcendental function. This implies that $L$ must be $L_{0}$ in this case.

Next we consider the case $c \neq 0$. We may assume that $c=1$ in this case. Using the same argument as above, we obtain a polynomial in $f$ of degree 4 which must vanish. Since $f$ is transcendental, all coefficients must vanish. From the coefficients of $f^{4}, f^{3}$ and $f^{2}$, we obtain the following relations

$$
\begin{gathered}
4 a^{3}-\tilde{g}_{2} a-\tilde{g}_{3}=0, \\
12 a^{2} b-4 b^{2}+4 a^{3} d+8 a b d-4 a^{2} d^{2}-b \tilde{g}_{2}-3 a d \tilde{g}_{2}-4 d \tilde{g}_{3}=0,
\end{gathered}
$$

and

$$
4 a b^{2}+4 a^{2} b d-b d \tilde{g}_{2}-a d^{2} \tilde{g}_{2}-2 d^{2} \tilde{g}_{3}=0 .
$$

From (3.17) and (3.19), we eliminate $\tilde{g}_{3}$. Then, noting that $a d-b \neq 0$, we have

$$
4 a b+d\left(8 a^{2}-\tilde{g}_{2}\right)=0 .
$$

(i) When $a \neq 0$, substituting $b=-d\left(8 a^{2}-\tilde{g}_{2}\right) /(4 a)$ from (3.20) and $\tilde{g}_{3}=4 a^{3}-\tilde{g}_{2} a$ from (3.17) into (3.18), we obtain $(a+d) d\left(12 a^{2}-\tilde{g}_{2}\right)^{2}=0$. We note that $d\left(12 a^{2}-\tilde{g}_{2}\right) \neq 0$. In fact, if $d\left(12 a^{2}-\tilde{g}_{2}\right)=0$, by (3.20) we obtain that $a=0$ since $a d-b \neq 0$, which is a contradiction. We have

$$
d=-a
$$

and from (3.20) we have

$$
b=\frac{8 a^{2}-\tilde{g}_{2}}{4} .
$$

(ii) When $a=0$, we have $\tilde{g}_{3}=0$ by (3.17) and $d \tilde{g}_{2}=0$ by (3.20). If $d \neq 0$, $\tilde{g}_{2}=0$. This implies that $27 \tilde{g}_{3}^{2}-\tilde{g}_{2}^{3}=0$, which is a contradiction. We have $d=0$. Substituting $a=0, d=0$ into (3.18), we obtain the equality $b\left(4 b+\tilde{g}_{2}\right)=0$. As $b \neq 0$ in this case $(a d-b \neq 0)$, we have $b=-\tilde{g}_{2} / 4$.

(i) and (ii) imply that (3.21) and (3.22) hold in any case.

By (3.17), we see that $a$ coincides with one of the roots of $G(x)=0$, say $e_{1}, e_{2}$ or $e_{3}$. We note that $\tilde{g}_{2}=-4\left(e_{1} e_{2}+e_{2} e_{3}+e_{3} e_{1}\right)$ and $\tilde{g}_{3}=4 e_{1} e_{2} e_{3}$. In view of (3.21) and (3.22), if $a=e_{1}$ then $b=e_{1}^{2}-e_{2}^{2}-e_{1} e_{2}$ and $d=-e_{1}$. This implies that $L$ coincides with $L_{1}$. Similarly we see that $L=L_{2}$ when $a=e_{2}$ and $L=L_{3}$ when $a=e_{3}$. 
PROOF OF THEOREM 3.1(i). Let $f$ and $g$ be two transcendental meromorphic solutions of (3.2) satisfying the hypothesis of this theorem. First we will show that $A(z)$ in (3.2) has no poles. From (3.2),

$$
A(z)=\frac{\left(f^{\prime}\right)^{2}}{G(f)}=\frac{\left(g^{\prime}\right)^{2}}{G(g)} \quad \text { and } \quad \frac{G(f)}{G(g)}=\left(\frac{f^{\prime}}{g^{\prime}}\right)^{2} .
$$

Suppose that $A$ has a pole $z_{0}$. From (3.23), there are four possibilities:

(i.1) $z_{0}$ is a pole of $f$ and a pole of $g$,

(i.2) $z_{0}$ is a pole of $f$ and a zero of $G(g)$,

(i.3) $z_{0}$ is a pole of $g$ and a zero of $G(f)$,

(i.4) $z_{0}$ is a zero of $G(f)$ and a zero of $G(g)$.

Here we make a remark. In the cases (i.2)-(i.4) we consider the zeros of $G(f)$ and $G(g)$. Assume that $z_{0}$ is a zero of $G(f)$. It follows that $f$ has one of the $e_{j}$ $(j=1,2,3)$ points at $z_{0}$. Without loss of generality we may assume that it is an $e_{1}$ point. We set $f_{1}=L_{1}(f)$, where $L_{1}$ is given in Remark 2 (B), that is,

$$
f_{1}=\frac{e_{1} f+e_{1}^{2}-e_{2}^{2}-e_{1} e_{2}}{f-e_{1}}
$$

Then we see by a simple computation that $f_{1}$ also satisfies (3.2) and $z_{0}$ is a pole of $f_{1}$. Hence the cases (i.2)-(i.4) reduce to the case (i.1), by using a suitable Möbius transformation which can be defined in a similar way to (3.24). Thus we have only to consider the case (i.1). Denote by $\mu_{A}, \mu_{f}$ and $\mu_{g}$ the orders of the poles at $z_{0}$ for $A, f$ and $g$.

From (3.2), we have $2\left(\mu_{f}+1\right)=3 \mu_{f}+\mu_{A}$, that is, $(1 \leq) \mu_{f}=2-\mu_{A}$. Hence $\mu_{f}=\mu_{A}=1$ and similarly $\mu_{g}=1$. Here we consider the Laurent expansions of $A, f$ and $g$ in a neighbourhood of $z_{0}$ as follows:

$$
\begin{array}{ll}
A(z)=\frac{R_{A}}{z-z_{0}}+\alpha_{A}+O\left(z-z_{0}\right), & R_{A} \neq 0, \\
f(z)=\frac{R_{f}}{z-z_{0}}+\alpha_{f}+O\left(z-z_{0}\right), & R_{f} \neq 0, \\
g(z)=\frac{R_{g}}{z-z_{0}}+\alpha_{g}+O\left(z-z_{0}\right), & R_{g} \neq 0 .
\end{array}
$$

From (3.11), $R_{f}=R_{g}=1 / 4 R_{A}$. Further, substituting these representations into (3.2) and comparing the coefficients of terms $\left(z-z_{0}\right)^{-3}$, we have

$$
\alpha_{f}=\alpha_{g}=\frac{-R_{f} \alpha_{A}}{3 R_{A}}=\frac{-\alpha_{A}}{12 R_{A}^{2}}
$$


By the assumption of this_lemma, the function $\varphi=f-g$ does not vanish identically, and by (3.25) $\varphi$ has a zero at $z_{0}$. However, by Lemma 3.4 it is impossible that $\varphi$ has a zero at $z_{0}$, a contradiction.

Secondly, we will show that all zeros of $A$ are of even order. Let $z_{1}$ be a zero of $A$. From (3.23), if $z_{1}$ is a zero of $f^{\prime}$ (respectively $g^{\prime}$ ) and if $z_{1}$ is not a zero of $G(f)$, (respectively $G(g)$ ), then the order of the zero of $A$ at $z_{1}$ is an even integer. Hence we shall consider the following four possibilities.

(i.5) $z_{1}$ is a pole of $f$ and a pole of $g$,

(i.6) $z_{1}$ is a pole of $f$, a zero of $g^{\prime}$ and a zero of $G(g)$,

(i.7) $z_{1}$ is a pole of $g$, a zero of $f^{\prime}$ and a zero of $G(f)$,

(i.8) $z_{1}$ a zero of $f^{\prime}$, a zero of $G(f)$, a zero of $g^{\prime}$ and a zero of $G(g)$.

We only have to treat the case (i.5). In fact, as in the cases (i.2)-(i.4) above, the cases (i.6)-(i.8) can be reduced to the case (i.5) by using suitable Möbius transformations. We denote by $\lambda$ the order of the zero of $A$ at $z_{1}$, and denote by $\mu_{f}$ and $\mu_{g}$ the orders of the poles of $f$ and $g$ at $z_{1}$.

In a manner similar to the proof of Lemma 3.4, we obtain $(1 \leq) \lambda=\mu_{f}-2=\mu_{g}-2$, which implies $\mu_{f} \geq 3$. As before, we write $\mu_{f}=\mu_{g}=\mu$.

Consider the Laurent expansions of $A, f$ and $g$ in a neighbourhood of $z_{1}$. Denote by $R_{A}$ the coefficient of $\left(z-z_{1}\right)^{\mu-2}$ in the expansion of $A$, and denote by $R_{f}, R_{g}$ the coefficients of $\left(z-z_{1}\right)^{-\mu}$ in the expansions of $f, g$ respectively. From (3.2), similarly to (3.11), we have

$$
R_{f}=R_{g}=\frac{\mu^{2}}{4 R_{A}} .
$$

We see that the coefficient of the term $\left(z-z_{1}\right)^{-2}$ in the right-hand side of (3.15) is $6 R_{A}\left(R_{f}+R_{g}\right)=3 \mu^{2}$ by $(3.26)$.

We divide the behaviour of $\varphi$ at $z=z_{1}$ into three cases, namely, $\varphi$ has a pole at $z_{1}, \varphi$ has a zero at $z_{1}$, or $\varphi$ does not have a pole nor a zero at $z_{1}$.

We first assume that $\varphi$ has a pole at $z_{1}$ of order $\nu$. Note that by (3.26) $\nu$ is at most $\mu-1$. In the left-hand side of (3.15), the coefficient of double pole $z_{1}$ is $v(\nu+1)+(\mu-2) \nu / 2=\nu^{2}+\mu \nu / 2$. Hence we have $2 \nu^{2}+\mu \nu-6 \mu^{2}=0$, so $\nu=-2 \mu$ or $2 v=3 \mu$. Since $\mu$ and $\nu$ are positive, $\nu=-2 \mu$ is absurd. If $2 \nu=3 \mu$ then since $v \leq \mu-1$, we have $\mu \leq-2$ which is also absurd.

Next we treat the case where $\varphi$ has a zero at $z_{1}$. By the assumption, the function $\varphi=f-g$ does not vanish. Hence, in view of Lemma 3.4, this case does not occur.

Finally we consider the case where $\varphi$ does not have a pole nor a zero at $z_{1}$. In this case $z_{1}$ is a simple pole or a regular point of the left-hand side of (3.15). However the right-hand side has a double pole, a contradiction.

Therefore $A$ must be a polynomial whose zeros are of even order, which implies that there exists a polynomial $a$ such that $A=\left(a^{\prime}\right)^{2}$. 
PROOF OF THEOREM 3.1(ii). We follow the idea in the proofs of Lemma 3.3 parts (a) and (b), (see Bank and Kaufman [2]). Let $f$ be a transcendental meromorphic solution of (3.2). We fix $z_{0} \in \mathbb{C}$ which is not a pole of $f$ satisfying the conditions $a^{\prime}\left(z_{0}\right) \neq 0$, $\wp^{\prime}\left(z_{0}\right) \neq 0$ and $f^{\prime}\left(z_{0}\right) \neq 0$ (or $G\left(f\left(z_{0}\right)\right) \neq 0$ ). Denote by $D_{0}$ a fundamental parallelogram of $\wp$ that contains $z_{0}$. Further we set $f\left(z_{0}\right)=b_{0}$ and $f^{\prime}\left(z_{0}\right) / a^{\prime}\left(z_{0}\right)=b_{1}$. Then from (3.2), $b_{1}^{2}=G\left(b_{0}\right)$. In view of Lemma 3.2, there exists $z_{1} \in D_{0}$ such that $\wp\left(z_{1}\right)=b_{0}$ and $\wp^{\prime}\left(z_{1}\right)=b_{1}$. We set $\alpha(z)=a(z)+z_{1}-a\left(z_{0}\right)$ and

$$
f_{1}=f_{1}(z)=\wp(\alpha(z))=\wp\left(a(z)+z_{1}-a\left(z_{0}\right)\right) .
$$

Then $f_{1}^{\prime}(z)=\wp^{\prime}(\alpha(z)) \alpha^{\prime}(z)=\wp^{\prime}(\alpha(z)) a^{\prime}(z)$ and hence

$$
\left(f_{1}^{\prime}\right)^{2}=\left(\wp^{\prime}(\alpha)\right)^{2}\left(a^{\prime}\right)^{2}=A G(\wp(\alpha))=A G\left(f_{1}\right)
$$

which implies that $f_{1}$ is a meromorphic solution of (3.2). We have that

$$
\begin{aligned}
& f_{1}\left(z_{0}\right)=\wp\left(a\left(z_{0}\right)+z_{1}-a\left(z_{0}\right)\right)=\wp\left(z_{1}\right)=b_{0}=f\left(z_{0}\right) \\
& f_{1}^{\prime}\left(z_{0}\right)=\wp^{\prime}\left(a\left(z_{0}\right)+z_{1}-a\left(z_{0}\right)\right) a^{\prime}\left(z_{0}\right)=\wp^{\prime}\left(z_{1}\right) a^{\prime}\left(z_{0}\right)=b_{1} a^{\prime}\left(z_{0}\right)=f^{\prime}\left(z_{0}\right) .
\end{aligned}
$$

Set $\psi=f-f_{1}$. Then from (3.27) and (3.28) we have that $\psi\left(z_{0}\right)=\psi^{\prime}\left(z_{0}\right)=0$. We see that $A^{\prime} / 2 A$ and $A$ are analytic at $z_{0}$ from our assumption. Regarding $g$ as $f_{1}$ and $\varphi$ as $\psi$ in (3.14), we conclude that $\psi=0$, so $f$ and $f_{1}$ must coincide. This proves (ii).

ProOF OF THEOREM 3.1(iii). Let $u$ and $v$ denote meromorphic solutions of (3.2) and let $a(z)$ be a polynomial given in (i). We may assume that $u=u(z)=\wp(a(z))$ and we can write $v=v(z)=\wp(a(z)+c)$ for a constant $c \in \mathbb{C}$ by (ii). Put $\wp(c)=d_{0}$ and $\wp^{\prime}(c)=d_{1}$. Then by the addition formula for the $\wp$-function,

$$
\wp(a(z)+c)=\frac{1}{4}\left(\frac{\wp^{\prime}(a(z))-\wp^{\prime}(c)}{\wp(a(z))-\wp(c)}\right)^{2}-\wp(a(z))-\wp(c),
$$

that is,

$$
v=\frac{1}{4}\left(\frac{\wp^{\prime}(a(z))-d_{1}}{u-d_{0}}\right)^{2}-u-d_{0} .
$$

Since $d_{1}^{2}=G\left(d_{0}\right)$ and $\left(a^{\prime}(z)\right)^{2}=A(z)$, from (3.2) and (3.29) we obtain

$$
\left(4\left(v+u+d_{0}\right)\left(u-d_{0}\right)^{2}-G(u)-G\left(d_{0}\right)\right)^{2}=4 G\left(d_{0}\right) G(u) .
$$

Put $U=U(z)=u(z)-d_{0}$ and $V=V(z)=v(z)-d_{0}$. Then since

$$
G\left(d_{0}\right)=4 d_{0}^{3}-\tilde{g}_{2} d_{0}-\tilde{g}_{3} \text { and } G^{\prime}\left(d_{0}\right)=12 d_{0}^{2}-\tilde{g}_{2},
$$

we can write (3.30) as

$$
U^{2} V^{2}-\frac{1}{2} G^{\prime}\left(d_{0}\right) U V-G\left(d_{0}\right)(U+V)+\frac{1}{16}\left(G^{\prime}\left(d_{0}\right)^{2}-48 d_{0} G\left(d_{0}\right)\right)=0,
$$


which confirms that $U$ and $V$ satisfy a relation of the form (3.4).

Conversely, we suppose that the relation (3.4) holds for meromorphic functions $U$ and $V$. We differentiate (3.4) to obtain

$$
U^{\prime}\left(2 U V^{2}-G_{2} V-G_{1}\right)=-V^{\prime}\left(2 V U^{2}-G_{2} U-G_{1}\right) .
$$

Using (3.4) we have

$$
\begin{aligned}
& \left(2 V U^{2}-G_{2} U-G_{1}\right)^{2} \\
& \quad=4 U^{2}\left(G_{2} U V+G_{1}(U+V)+G_{0}\right)+G_{2}^{2} U^{2}+G_{1}^{2}-4 U^{3} V G_{2}-4 U^{2} V G_{1} \\
& \quad+2 G_{2} G_{1} U=4 G_{1} U^{3}+\left(4 G_{0}+G_{2}^{2}\right) U^{2}+2 G_{1} G_{2} U+G_{1}^{2}=G_{1} K(U) .
\end{aligned}
$$

Similarly we obtain

$$
\left(2 U V^{2}-G_{2} V-G_{1}\right)^{2}=G_{1} K(V) .
$$

Combining (3.31)-(3.33), we obtain the assertion (3.5) with (3.6).

PROOF OF COROLlaRY 3.2. (a) This assertion follows from (ii) of Theorem 3.1.

(b) Suppose that (3.2) has a transcendental meromorphic solution $f$. If there exists attranscendental meromorphic solution $g$ of (3.2) such that $g \neq L(f)$ for some Möbius transformation then we have $\# \mathfrak{I}(A)=\infty$ by (a). For the proof of (b), it remains to find the number of Möbius transformations $L_{j}$ such that $L_{j}(f)$ satisfies the equation (3.2) if $\# \mathfrak{I}(A) \neq 0, \infty$. By means of Remark 2(B), the number of such Möbius transformations is equal to four, so $\# \mathfrak{T}(A)=4$.

(c) In the case $f=L(g)$ for a Möbius transformation $L$, we have, by means of Nevanlinna's first fundamental theorem, $T(r, f)=T(r, g)+O(1)$. We may suppose that $f \neq L(g)$ for any Möbius transformation $L$. Then in view of Theorem 3.1(iii), there is a $d_{0} \in \mathbb{C}$ such that the functions $f_{0}=f-d_{0}$ and $g_{0}=g-d_{0}$ satisfy an algebraic relation (3.4). Since $T\left(r, f_{0}\right)=T(r, f)+O(1)$ and $T\left(r, g_{0}\right)=T(r, g)+O(1)$, we need only show that $f_{0}$ and $g_{0}$ satisfy the assertion of part (c), namely that $T\left(r, f_{0}\right)=T\left(r, g_{0}\right)+O(1)$. If $G_{1}=0$ in (3.4) then $f_{0} g_{0}$ is a constant, from which we obtain that $T\left(r, f_{0}\right)=T\left(r, g_{0}\right)+O(1)$. In what follows, we assume that $G_{1} \neq 0$. Define meromorphic functions

$$
f_{1}=-\frac{G_{1} g_{0}+G_{0}}{f_{0} g_{0}^{2}} \text { and } g_{1}=-\frac{G_{1} f_{0}+G_{0}}{g_{0} f_{0}^{2}} .
$$

From (3.3) for $U=f_{0}, V=g_{0}$, we have

$$
f_{0}-\frac{G_{2} g_{0}+G_{1}}{g_{0}^{2}}=\frac{G_{1} g_{0}+G_{0}}{f_{0} g_{0}^{2}}
$$


Eliminating $f_{0}$ from this equation by using the first one of (3.33), we see that $f_{1}$ and $g_{0}$ satisfy (3.4). Similarly we see that $f_{0}$ and $g_{1}$ satisfy (3.4), so

$$
\begin{aligned}
& f_{1}^{2} g_{0}^{2}-G_{2} f_{1} g_{0}-G_{1}\left(f_{1}+g_{0}\right)-G_{0}=0, \\
& f_{0}^{2} g_{1}^{2}-G_{2} f_{0} g_{1}-G_{1}\left(f_{0}+g_{1}\right)-G_{0}=0 .
\end{aligned}
$$

Thus $f_{0}, g_{0}, f_{1}$ and $g_{1}$ are transcendental meromorphic solutions of

$$
\left(w^{\prime}\right)^{2}=A(z) K(w),
$$

where $A(z)$ is given in (3.2) and $K(w)$ is given in (3.6). We also have

$$
f_{0}+f_{1}=\frac{G_{2} g_{0}+G_{1}}{g_{0}^{2}} \text { and } g_{0}+g_{1}=\frac{G_{2} f_{0}+G_{1}}{f_{0}^{2}} \text {. }
$$

It follows from (3.38) and $G_{1} \neq 0$ that

$$
2 T\left(r, g_{0}\right) \leq T\left(r, f_{0}\right)+T\left(r, f_{1}\right)+O(1) .
$$

Using (3.34) and (3.38), we obtain

$$
\frac{1}{f_{0}}+\frac{1}{f_{1}}=-\frac{G_{2} g_{0}+G_{1}}{G_{1} g_{0}+G_{0}} .
$$

By means of Nevanlinna's first fundamental theorem of and (3.40),

$$
T\left(r, f_{1}\right) \leq T\left(r, g_{0}\right)+T\left(r, f_{0}\right)+O(1) .
$$

Combining (3.39) and (3.41), we have $T\left(r, g_{0}\right) \leq 2 T\left(r, f_{0}\right)+O(1)$. Changing the roles of $f_{0}(z)$ and $g_{0}(z)$, we obtain $T\left(r, f_{0}\right) \leq 2 T\left(r, g_{0}\right)+O(1)$. This implies that if $\varphi(r)=S\left(r, f_{0}\right)$, then $\varphi(r)=S\left(r, g_{0}\right)$, and if $\varphi(r)=S\left(r, g_{0}\right)$, then $\varphi(r)=S\left(r, f_{0}\right)$. Hence for two meromorphic functions $f$ and $g$, we can write $S(r, f)=S(r)$ and $S(r, g)=S(r)$.

We recall some properties of a transcendental meromorphic solution $w(z)$ of (3.37). Let $w(z)$ be a transcendental meromorphic solution of (3.37). Then, by means of Gol'dberg's theorem [4], we see that $w(z)$ is of finite order. We also have that all poles of $w(z)$, except for a finite number, are double, and $m(r, w)=O(\log r)$. Also all zeros of $w(z)$, except for a finite number, are simple, and $m(r, 1 / w)=O(\log r)$ since we assume $G_{1} \neq 0$. Hence,

$$
\begin{aligned}
N(r, w) & =2 \bar{N}(r, w)+O(\log r)=T(r, w)+O(\log r), \quad \text { and } \\
N(r, 1 / w) & =\bar{N}(r, 1 / w)+O(\log r)=T(r, w)+O(\log r) .
\end{aligned}
$$

Let $z_{0}$ be a pole of $f_{0}(z)$ and let $z_{1}$ be a pole of $f_{1}(z)$. Then we see from (3.4) and (3.35) (or (3.34)) that $z_{0}$ is a zero of $g_{0}$ and $z_{1}$ is also a zero of $g_{0}$. If both $f_{0}(z)$ 
and $f_{1}(z)$ have a common double pole $z_{2}$ then $z_{2}$ is a zero of $g_{0}(z)$ of multiplicity at least two. From (3.43), the counting function of such common poles is $O(\log r)$. Thus it follows that

$$
\bar{N}\left(r, f_{0}\right)+\bar{N}\left(r, f_{1}\right) \leq \bar{N}\left(r, 1 / g_{0}\right)+O(\log r) .
$$

From (3.42)-(3.44),

$$
T\left(r, f_{0}\right)+T\left(r, f_{1}\right) \leq 2 T\left(r, g_{0}\right)+O(\log r) .
$$

Combining this with (3.39), we obtain

$$
T\left(r, f_{0}\right)+T\left(r, f_{1}\right)=2 T\left(r, g_{0}\right)+O(\log r) .
$$

Further we define

$$
g_{2}=-\frac{G_{1} f_{1}+G_{0}}{g_{0} f_{1}^{2}} \text { and } f_{2}=-\frac{G_{1} g_{1}+G_{0}}{f_{0} g_{1}^{2}} .
$$

Repeating this process, we define sequences of meromorphic functions $f_{0}, g_{1}, f_{2}$, $g_{3}, \ldots$, and $g_{0}, f_{1}, g_{2}, f_{3}, \ldots$. Namely, for $k=0,1,2, \ldots$, we set

$$
\begin{aligned}
& f_{2 k+3}=-\frac{G_{1} g_{2 k+2}+G_{0}}{f_{2 k+1} g_{2 k+2}^{2}}, \quad g_{2 k+2}=-\frac{G_{1} f_{2 k+1}+G_{0}}{g_{2 k} f_{2 k+1}^{2}}, \\
& g_{2 k+3}=-\frac{G_{1} f_{2 k+2}+G_{0}}{g_{2 k+1} f_{2 k+2}^{2}}, \quad f_{2 k+2}=-\frac{G_{1} g_{2 k+1}+G_{0}}{f_{2 k} g_{2 k+1}^{2}} .
\end{aligned}
$$

Then we see that all the functions

$$
\left\{f_{j}(z)\right\}(j=0,1, \ldots) \quad \text { and }\left\{g_{k}(z)\right\} \quad(k=0,1, \ldots)
$$

are transcendental and satisfy the differential equation (3.37), and all pairs

$$
\left(f_{j}(z), g_{j+1}(z)\right) \quad \text { and }\left(g_{j}(z), f_{j+1}(z)\right) \quad(j=0,1, \ldots)
$$

satisfy (3.4) and all triples

$$
\left(f_{j-1}(z), f_{j+1}(z), g_{j}(z)\right) \quad \text { and } \quad\left(g_{j-1}(z), g_{j+1}(z), f_{j}(z)\right) \quad(j=1,2, \ldots)
$$

satisfy (3.45). For $j=0,1,2, \ldots$, we write

$$
h_{j}(z)= \begin{cases}f_{j}(z), & \text { if } j \text { is odd } \\ g_{j}(z), & \text { if } j \text { is even. }\end{cases}
$$

Let $a_{0}, b_{0}, a_{1}$ and $b_{1}$ be positive constants. We assume that there exists a sequence $\left\{r_{n}\right\}$ such that $r_{n} \rightarrow \infty$ as $n \rightarrow \infty$ and

$$
\begin{aligned}
& T\left(r_{n}, h_{0}\right) \leq a_{0} T\left(r_{n}, f_{0}\right)+O\left(\log r_{n}\right) \\
& T\left(r_{n}, h_{0}\right) \geq b_{0} T\left(r_{n}, f_{0}\right)+O\left(\log r_{n}\right)
\end{aligned}
$$

and 


$$
\begin{aligned}
& T\left(r_{n}, h_{1}\right) \leq a_{1} T\left(r_{n}, f_{0}\right)+O(\log r) \\
& T\left(r_{n}, h_{1}\right) \geq b_{1} T\left(r_{n}, f_{0}\right)+O(\log r) .
\end{aligned}
$$

We assert that there exist sequences $\left\{a_{j}\right\}$ and $\left\{b_{j}\right\}, j=0,1,2, \ldots$, such that

$$
\begin{aligned}
& T\left(r_{n}, h_{j}\right) \leq a_{j} T\left(r_{n}, f_{0}\right)+O\left(\log r_{n}\right) \quad \text { and } \\
& T\left(r_{n}, h_{j}\right) \geq b_{j} T\left(r_{n}, f_{0}\right)+O\left(\log r_{n}\right) .
\end{aligned}
$$

In view of (3.45) and the comment that we made after the definitions of $\left\{f_{j}(z)\right\}$ and $\left\{g_{j}(z)\right\}$, we have, for $j=1,2, \ldots$,

$$
T\left(r_{n}, h_{j-1}\right)+T\left(r_{n}, h_{j+1}\right)=2 T\left(r_{n}, h_{j}\right)+O\left(\log r_{n}\right) .
$$

Assume that (3.48) and (3.49) hold for $j=0,1,2, \ldots, k$. Then from (3.50),

$$
\begin{aligned}
T\left(r_{n}, h_{k+1}\right) & =2 T\left(r_{n}, h_{k}\right)-T\left(r_{n}, h_{k-1}\right)+O\left(\log r_{n}\right) \\
& \leq 2 a_{k} T\left(r_{n}, f_{0}\right)-b_{k-1} T\left(r_{n}, f_{0}\right)+O\left(\log r_{n}\right),
\end{aligned}
$$

which gives

$$
a_{k+1}=2 a_{k}-b_{k-1}
$$

Similarly, we obtain

$$
b_{k+1}=2 b_{k}-a_{k-1} .
$$

Therefore, using the assumptions (3.46) and (3.47), we can use (3.51) and (3.52) recursively to obtain a sequence $\left\{a_{n}\right\}$ which satisfies (3.48) and a sequence $\left\{b_{n}\right\}$ which satisfies (3.49).

We now compute $a_{k}$ and $b_{k}$ concretely. Put $c_{k}=a_{k}+b_{k}$. Then we have $c_{k+1}-2 c_{k}+c_{k-1}=0$ and hence $c_{k}=\left(c_{1}-c_{0}\right) k+c_{0}, k=0,1,2, \ldots$ Thus we obtain

$$
a_{k+1}-2 a_{k}-a_{k-1}=\mu k+\nu,
$$

where $\mu=c_{0}-c_{1}$ and $v=c_{1}-2 c_{0}$. In (3.53), we set $d_{k}=a_{k+1}-a_{k}$. Then we have $d_{k}-2 d_{k-1}-d_{k-2}=\mu$. Further, we put $e_{k}=d_{k}+\mu / 2$. Then

$$
e_{k}-2 e_{k-1}-e_{k-2}=0 \text {. }
$$

Thus we can write $e_{k}$ with some constants $\gamma_{1}$ and $\gamma_{2}$ as

$$
e_{k}=\gamma_{1} \lambda_{1}^{k}+\gamma_{2} \lambda_{2}^{k}
$$

where $\lambda_{1}=1+\sqrt{2}$ and $\lambda_{2}=1-\sqrt{2}$, (which are the roots of $t^{2}-2 t-1=0$ ), 
see, for example, [3]. Thus $d_{k}=e_{k}-\mu / 2$ and hence for $k=1,2, \ldots$, we have

$$
\begin{aligned}
a_{k} & =\sum_{j=0}^{k-1} d_{j}+a_{0}=\sum_{j=0}^{k-1}\left(e_{j}-\frac{\mu}{2}\right)+a_{0}=\sum_{j=0}^{k-1}\left(\gamma_{1} \lambda_{1}^{j}+\gamma_{2} \lambda_{2}^{j}-\frac{\mu}{2}\right)+a_{0} \\
& =\gamma_{1} \frac{1-\lambda_{1}^{k}}{1-\lambda_{1}}+\gamma_{2} \frac{1-\lambda_{2}^{k}}{1-\lambda_{2}}-\frac{\mu}{2} k+a_{0}, \quad \text { and } \\
b_{k} & =2 a_{k}-a_{k+1} \\
& =\frac{\gamma_{1}}{1-\lambda_{1}}\left(1-2 \lambda_{1}^{k}+\lambda_{1}^{k+1}\right)+\frac{\gamma_{2}}{1-\lambda_{2}}\left(1-2 \lambda_{2}^{k}+\lambda_{2}^{k+1}\right)-\frac{\mu}{2}(k-1)+a_{0} .
\end{aligned}
$$

We assert that

$$
\liminf _{r \rightarrow \infty} \frac{T\left(r, f_{1}\right)}{T\left(r, f_{0}\right)} \geq 1 \text { and } \liminf _{r \rightarrow \infty} \frac{T\left(r, g_{1}\right)}{T\left(r, g_{0}\right)} \geq 1
$$

To show this, we assume that

$$
\liminf _{r \rightarrow \infty} \frac{T\left(r, f_{1}\right)}{T\left(r, f_{0}\right)}=\alpha<1
$$

For any $\epsilon>0$ such that $\alpha+\epsilon<1$, there exists a sequence $\left\{r_{n}\right\}=\left\{r_{n}(\epsilon)\right\}$ satisfying

$$
T\left(r_{n}, f_{1}\right) \leq(\alpha+\epsilon) T\left(r_{n}, f_{0}\right) \text { and } T\left(r_{n}, f_{1}\right) \geq(\alpha-\epsilon) T\left(r_{n}, f_{0}\right),
$$

for $n \geq n_{0}(\epsilon)$. Later we choose a suitable $\epsilon$. From (3.45),

Similarly, we have

$$
\begin{aligned}
T\left(r_{n}, h_{0}\right) & =T\left(r_{n}, g_{0}\right)=\frac{T\left(r_{n}, f_{0}\right)+T\left(r_{n}, f_{1}\right)}{2}+O\left(\log r_{n}\right) \\
& \leq \frac{T\left(r_{n}, f_{0}\right)+(\alpha+\epsilon) T\left(r_{n}, f_{0}\right)}{2}+O\left(\log r_{n}\right) \\
& =\frac{(1+\alpha+\epsilon) T\left(r_{n}, f_{0}\right)}{2}+O\left(\log r_{n}\right) .
\end{aligned}
$$

$$
T\left(r_{n}, h_{0}\right) \geq \frac{(1+\alpha-\epsilon) T\left(r_{n}, f_{0}\right)}{2}+O\left(\log r_{n}\right) .
$$

We now set

$$
a_{0}=\frac{1+\alpha+\epsilon}{2}, \quad b_{0}=\frac{1+\alpha-\epsilon}{2}, \quad a_{1}=\alpha+\epsilon, \quad \text { and } \quad b_{1}=\alpha-\epsilon .
$$

We compute $\mu, \nu, \gamma_{1}$ and $\gamma_{2}$ concretely under our assumptions. We have

$$
\mu=c_{0}-c_{1}=\left(a_{0}+b_{0}\right)-\left(a_{1}+b_{1}\right)=1-\alpha \text { and } \nu=c_{1}-2 c_{0}=-2 \text {. }
$$


From (3.53),

$$
a_{2}=2 a_{1}+a_{0}+\mu+\nu=\frac{3}{2} \alpha+\frac{5}{2} \epsilon-1 / 2 .
$$

On the other hand, from (3.56),

Hence we have

$$
\begin{aligned}
& a_{1}=\gamma_{1}+\gamma_{2}+\alpha+\frac{\epsilon}{2} \\
& a_{2}=\left(1+\lambda_{1}\right) \gamma_{1}+\left(1+\lambda_{2}\right) \gamma_{2}+\frac{3}{2} \alpha+\frac{1}{2} \epsilon-\frac{1}{2} .
\end{aligned}
$$

$$
\gamma_{1}+\gamma_{2}=\frac{\epsilon}{2} \text { and }\left(1+\lambda_{1}\right) \gamma_{1}+\left(1+\lambda_{2}\right) \gamma_{2}=2 \epsilon .
$$

Since $\lambda_{1}=1+\sqrt{2}$ and $\lambda_{2}=1-\sqrt{2}$, we obtain

$$
\gamma_{1}=\frac{(1+\sqrt{2})}{4} \epsilon, \quad \gamma_{2}=\frac{(1-\sqrt{2})}{4} \epsilon .
$$

Hence we can write

$$
\begin{aligned}
& a_{k}=(\alpha-1) k+\frac{1+\alpha+\epsilon}{2} \\
& \quad+\epsilon\left(\left(\frac{1+\sqrt{2}}{4}\right) \frac{(1+\sqrt{2})^{k}-1}{\sqrt{2}}+\left(\frac{1-\sqrt{2}}{4}\right) \frac{1-(1-\sqrt{2})^{k}}{\sqrt{2}}\right) .
\end{aligned}
$$

Since we assume that $\alpha<1$, we can take $k=k(\alpha)$ so large that $(\alpha-1) k+1<0$. Once we find such a $k$, we fix it. Then we choose $\epsilon$ so small that $a_{k}<0$. For this $\epsilon$, there exists $\left\{r_{n}\right\}=\left\{r_{n}(\epsilon)\right\}$ satisfying (3.59), in particular,

$$
T\left(r_{n}, h_{j}\right) \leq a_{k} T\left(r_{n}, f_{0}\right)+O\left(\log r_{n}\right) .
$$

We observe the term $O\left(\log r_{n}\right)$ in (3.61). Write this term as $\psi\left(\log r_{n}\right)$. Then the function $\psi(x)$ in $x$ depends on $k$. However, it is independent of $\epsilon$. Since $h_{0}$ is transcendental and $a_{k}<0$, the right hand side of (3.61) is negative for sufficiently large $n$, a contradiction. This gives the first inequality in (3.57). On the other hand, we consider a sequence of functions

$$
h_{j}^{*}(z)= \begin{cases}f_{j}(z), & \text { if } j \text { is even } \\ g_{j}(z), & \text { if } j \text { is odd }\end{cases}
$$

instead of $h_{j}(z)$ above. Then we obtain the second inequality in (3.57) by similar arguments. Hence the assertion (3.57) follows. It follows from (3.45) and the first inequality in (3.57) that

$$
\liminf _{r \rightarrow \infty} \frac{T\left(r, g_{0}\right)}{T\left(r, f_{0}\right)} \geq 1
$$


We recall the remark that we posed after the definitions of $\left\{f_{j}(z)\right\}$ and $\left\{g_{j}(z)\right\}$, in particular,

$$
T\left(r, g_{0}\right)+T\left(r, g_{1}\right)=2 T\left(r, f_{0}\right)+O(\log r) .
$$

From this and the second inequality in (3.57), we have

$$
\liminf _{r \rightarrow \infty} \frac{T\left(r, f_{0}\right)}{T\left(r, g_{0}\right)} \geq 1
$$

and hence

$$
\limsup _{r \rightarrow \infty} \frac{T\left(r, g_{0}\right)}{T\left(r, f_{0}\right)} \leq 1 \text {. }
$$

Hence we see that $\lim _{r \rightarrow \infty} T\left(r, f_{0}\right) / T\left(r, g_{0}\right)=1$. This implics that as $r \rightarrow \infty$, $T\left(r, f_{0}\right)=(1+o(1)) T\left(r, g_{0}\right)$ which gives (3.7). Finally, we comment that the case $h_{j}(z)=h_{i}(z)$ for some $j \neq i$ is included in our arguments. We have thus proved (c).

\section{Examples}

Finally, we state some examples in this section. As mentioned in the statement in Theorem 2.3, a condition that gives $\# \mathfrak{S}(A) \geq 3$ is obtained and in Remark 1 , a

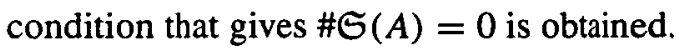

A natural question arises: Under what conditions does $\# \mathfrak{S}(A)=2$ occur?

We shall give some examples of $A$ in (2.1) for which $\# \mathbb{S}(A)=2$ and an example for (3.2) having the property $\# \mathfrak{I}(A)=4$.

EXAMPLE 1. $\mathfrak{S}\left(\frac{1}{4 z}\right)=\{\cosh \sqrt{z},-\cosh \sqrt{z}\}$.

In fact, it is easy to see that $\cosh \sqrt{z}$ and $-\cosh \sqrt{z}$ are transcendental entire solutions of the differential equation

$$
\left(f^{\prime}\right)^{2}=\frac{1}{4 z}\left(f^{2}-1\right)
$$

It follows from Theorem 2.3 (i) that there is no other solution to the equation above.

Similarly let $p(z)$ be a polynomial with simple zeros only. Then,

$$
\begin{aligned}
\mathfrak{S}\left(p\left(p^{\prime}\right)^{2}\right) & =\left\{ \pm \cosh \left(\frac{2}{3} p^{3 / 2}\right)\right\}, \\
\mathfrak{S}\left(\frac{\left(p^{\prime}\right)^{2}}{p}\right) & =\left\{ \pm \cosh 2 p^{1 / 2}\right\}, \\
\mathfrak{S}\left(4\left(z^{2}-1\right)\right) & =\left\{ \pm \cosh \left(z \sqrt{z^{2}-1}-\log \left(z+\sqrt{z^{2}-1}\right)\right)\right\} .
\end{aligned}
$$


EXAMPLE 2. The equation

$$
\left(f^{\prime}\right)^{2}=\frac{1}{4 z}\left(4 f^{3}-\tilde{g}_{2} f-\tilde{g}_{3}\right)
$$

possesses a solution $\wp(\sqrt{z})$, where $\wp$ is Weierstrass' elliptic function satisfying (3.1). Clearly $\wp(\sqrt{z}+c), c \neq 0 \in \mathbb{C}$, is not meromorphic and hence \#T $(1 / 4 z)=4$.

\section{Acknowledgements}

We would like to thank Professor Kazunari Sawada for his valuable suggestions. We would also like to thank the referee for his/her valuable suggestions for improving the paper.

\section{References}

[1] S. B. Bank, G. G. Gundersen and I. Laine, 'Meromorphic solutions of the Riccati differential equation', Ann. Acad. Sci. Fenn. Ser. A I Math. 6 (1981), 369-398.

[2] S. B. Bank and R. P. Kaufman, 'On the growth of meromorphic solutions of the differential equation $\left(y^{\prime}\right)^{m}=r(z, y)$ ', Acta Math. 144 (1980), 223-248.

[3] S. Elaydi, An Introduction to Difference Equations (Springer-Verlag, New York, 1996).

[4] A. A. Gol'dberg, 'On single-valued solutions of first-order differential equations', Ukrain. Mat. Zh. 8 (1956), 254-261.

[5] F. Gross, 'On the equation $f^{n}+g^{n}=1$ ', Bull. Amer. Math. Soc. 72 (1966), 86-88.

[6] W. K. Hayman, Meromorphic Functions (Oxford at the Clarendon Press, 1964).

[7] Y. Z. He and I. Laine, 'The Hayman-Miles theorem and the differential equation $\left(y^{\prime}\right)^{n}=r(z, y)$ ', Analysis (Munich) 10 (1990), 387-396.

[8] H. Herold, Differentialgleichungen im Komplexen (Vandenhoeck \& Ruprecht, Gottingen, 1975).

[9] E. Hille, Ordinary Differential Equations in the Complex Domain (Wiley and Sons, New YorkLondon-Sydney-Toronto, 1976).

[10] I. Laine, 'On the behavior of the solution of some first order differential equations', Ann. Acad. Sci. Fenn. Ser. A I 497 (1971).

[11] _-, Nevanlinna Theory and Complex Differential Equations (W. Gruyter, Berlin-New York, 1992).

[12] R. Nevanlinna, Le théorème de Picard-Borel et la théorie des fonctions méromorphes (GauthierVillard, Paris, 1929).

[13] N. Steinmetz, Eigenschaften eindeutiger Lösungen gewöhnlicher Differentialgleichungen im Komplexen (Ph.D. Thesis, Karlsruhe, 1978).

[14] _ - 'Zur theorie der binomischen differentialgleichungen', Math. Ann. 5 (1979), 263-274.

[15] G. Valiron, 'Sur la dérivée des fonctions algéroïdes', Bull. Soc. Math. France 59 (1931), 17-39.

[16] J. von Rieth, Untersuchungen gewisser Klassen gewöhnlicher Differentialgleichungen erster und zweiter Ordnung im Komplexen (Ph.D. Thesis, Technischen Hochschule, Aachen, 1986).

[17] K. Yosida, 'A generalization of Malmquist's theorem', Japan J. Math. 9 (1933), 253-256. 
Department of Mathematics

Nippon Institute of Technology

4-1 Gakuendai Miyashiro,

Minamisaitama Saitama 345-8501,

Japan

e-mail: ishi@nit.ac.jp
Center for General Education Aichi Institute of Technology Yakusa, Toyota-shi Aichi-ken 470-0392, Japan e-mail: toda3-302@coral.ocn.ne.jp 